\title{
21
}

\section{Observational Strategies from the Micro- to Mesoscale}

\author{
Jean-Louis Brenguier ${ }^{1}$ and Robert Wood $^{2}$ \\ ${ }^{1}$ Meteo-France, CNRS, Toulouse, France \\ ${ }^{2}$ Department of Atmospheric Sciences, University of Washington, Seattle, WA, U.S.A.
}

\begin{abstract}
In a changing climate, clouds are perturbed through large-scale variations in the general circulation induced by both greenhouse gases and aerosols. Some aerosols perturb cloud microphysical properties as well. The challenge for micro- and mesoscale observational studies of perturbed clouds is thus to establish the links between these two contrasting forcings, in an effort to understand how clouds respond to changes in the general circulation and to quantify how this response might be modulated by changes in their microphysical properties. The two generic classes of micro- to mesoscale observational strategies, the Eulerian column closure and the Lagrangian cloud system evolution approaches, are described using examples of low-level cloud studies, and recommendations are made on how they should be combined with large-scale information to address this issue.
\end{abstract}

\section{Introduction}

There is an apparent paradox in cloud physics: clouds are very diverse, in terms of morphology, depth, cloud base and top heights, horizontal extent, dynamics and microphysics; they exert very different radiative impacts, in terms of the balance between their albedo and their greenhouse effect, as well as very distinct dynamic impacts, in terms of how they contribute to the redistribution of water vapor, sensible, and latent heat in the atmosphere. Cloud spatial and temporal distributions are very heterogeneous; large regions may be devoid of clouds, whereas others can be overcast almost all year round. The lifetimes of cloud systems vary enormously from those that are almost stationary for days to others that are fleeting. Nevertheless - and herein lies the paradox - together they manage to maintain a somewhat constant Earth 
albedo, close to 0.3 , and a fairly constant global balance between their albedo and greenhouse contributions to the climate system.

Such a large-scale equilibrium calls for observational approaches that begin on a global scale and progress down to the microscale to capture the processes responsible for the regulation of the hydrological cycle. However, to date, most observational studies of clouds have focused on the microscale, progressing up to the mesoscale. Such studies have served as our fundamental tools for constructing, from the bottom-up, the current cloud models we use to simulate the climate system. However, model comparison exercises, such as those by the IPCC (2007), clearly show that some key feedback processes are still poorly represented. Moreover, although the models predict a mean cloud radiative forcing in agreement with observations, they also exhibit noticeable biases, with a consistent overprediction of optically thick clouds and an underprediction of optically thin low and middle-top clouds (Zhang et al. 2005). This led the IPCC to conclude that "differences in cloud response are the primary source of inter-model differences in climate sensitivity" (Randall et al. 2007, p. 633).

Better understanding of how clouds react to anthropogenic forcings is therefore a priority for improving the accuracy of climate change projections. Anthropogenic forcings, however, can produce very diverse impacts on the general circulation. The primary greenhouse gases have a long residence time (centuries) and are homogeneously distributed. Aerosols, by contrast, have a short residence time (days to weeks) and, consequently, their spatial distribution is heterogeneous, mainly concentrated in the vicinity of the sources. Greenhouse gases interact primarily with longwave radiation. The net radiative impact of aerosols depends on their chemical composition and the balance between their light scattering and absorbing contributions. Impacts of greenhouse gases on clouds can thus be explored from a global perspective to examine, for instance, how the increase in the column water vapor might be compensated by a damping of the convective mass flux to reduce the fractional increase in precipitation (Held and Soden 2006). Aerosol impacts should also be considered at the regional scale, where they are concentrated. The hypothesized "elevated heat pump" effect (Lau et al. 2006) provides an example of a plausible, but currently unverified, regional-scale aerosol effect which may impact the Indian monsoon at the Himalaya foothills.

This greenhouse perspective suggests that anthropogenic forcings might only perturb clouds by modifying the general circulation (i.e., from the global scale down to the cloud scale). However, aerosols impact also cloud microphysics, since some act as droplet or ice crystal nuclei. Such microphysical changes can propagate up to the cloud scale. The first order response to changing nucleus concentration is an effect upon the cloud albedo by changing the surface area of the droplets, but feedbacks on cloud-scale dynamics must also be considered to understand the response fully. By modifying cloud microphysical and optical properties, aerosol particles thus perturb clouds not only from the global/regional scale downward, via their direct effect, but also upward 
from the microscale (the aerosol indirect effect). Parameterizations of these processes in global climate models (GCMs) are very crude, partly because they involve nonlinear processes at scales that are not accessible to such models, but also because our knowledge of the various feedbacks of cloud microphysics on cloud dynamics is still limited. For example, high-resolution cloud models suggest that the aerosol impacts result in nontrivial effects, inducing either a decrease or an increase of the cloud liquid water path (LWP), with the sign of the response depending upon poorly understood factors. Hence, the aerosol impacts on clouds remain the most uncertain of the climate forcings in terms of efficacy (Forster et al. 2007, Fig. 2.19).

To improve climate change projections, it is crucial to understand how climate change might impact the spatiotemporal distribution and hence radiative forcing of clouds (from the global scale down). In addition, we must also find out whether clouds respond only to changes in large-scale dynamic forcings, or if microphysical processes might also modulate their response, thereby impacting the hydrological cycle and general circulation (i.e., from the bottom up).

Because of the very large range of scales involved, it has been difficult to connect large-scale observational studies of the hydrological cycle with microand mesoscale observations of cloud physics. Using examples from existing and future field studies, we will show how effective the micro- to mesoscale approach was for understanding cloud dynamics and microphysics, and will suggest that it now needs to evolve to progressively larger scales. This mandates a greater degree of multidisciplinarity in the design of future observational studies, in an effort to clarify the interactions between aerosol physics and chemistry, small-scale turbulent dynamics, radiation, and the hydrological cycle at the global scale.

\section{The Ingredients of an Observational Strategy}

To begin to determine the magnitude of the aforementioned potential cloud perturbations, the overall objective of the observational approach must be to quantify the respective susceptibilities of cloudiness or, more specifically, cloud radiative properties, $\delta c$, to changes in the general circulation and to internal microphysical changes induced by the aerosol. This approach is presented in Stevens and Brenguier (this volume) as:

$$
\delta c=\left(\frac{\partial c}{\partial M}\right)_{A}\left\{\left(\frac{\partial M}{\partial A}\right)_{G} \delta A+\left(\frac{\partial M}{\partial G}\right)_{A} \delta G\right\}+\left(\frac{\partial c}{\partial A_{\mu}}\right)_{M} \delta A_{\mu},
$$

where $c$ represents cloudiness, $M$ is the large-scale meteorology, $G$ stands for greenhouse gases, and $A$ for aerosols. $A_{\mu}$ specifically refers to the subset of the aerosols that may impact cloud microphysical properties, namely cloud condensation and ice nuclei (CCN, IN) and absorbing aerosol particles scavenged in hydrometeors. 
The term in the braces is the large-scale meteorological forcing, $\delta M$, related to the aerosol and greenhouse gas forcings, both of which are the result of integrated radiative forcings on large spatial scales. Thus, from Equation 21.1 we can represent cloud perturbations as:

$$
\begin{aligned}
& \delta c=\left(\frac{\partial c}{\partial A_{\mu}}\right)_{M} \delta A_{\mu}+\left(\frac{\partial c}{\partial M}\right)_{A} \delta M, \\
& \delta c=\lambda_{A_{\mu}} \delta A_{\mu}+\lambda_{M} \delta M,
\end{aligned}
$$

where $\lambda_{A \mu}$ and $\lambda_{M}$ are the sensitivities (susceptibilities) of clouds to perturbations in $A_{\mu}$ and $M$, respectively.

In designing a strategy for an observational program to assess the impacts of aerosols upon clouds, it is thus essential to ask whether variability in observed cloud radiative properties, $\delta c$, will be dominated by variability in aerosols or by the varying meteorological forcing. Equation 21.2 shows clearly that to determine the sensitivity of clouds to aerosol perturbations $\lambda_{A \mu}$ through observations, we must first understand the impacts of meteorology upon the cloud system, as expressed by $\lambda_{M}$.

The meteorological sensitivity, $\lambda_{M}$, is poorly known in many cases, and thus it is a major challenge to use observations to determine it. For marine stratocumulus clouds, there has been considerable success in relating cloud properties (e.g., cloud fractional coverage) to the large-scale meteorology using observations (e.g., Klein and Hartmann 1993; Klein et al. 1995; Wood and Bretherton 2006). As Stevens and Brenguier demonstrate (this volume), the meteorological sensitivity is very high in many cases, and this will limit our ability to attribute perturbations in clouds to those in aerosols.

We can further quantify the uncertainties in determining the sensitivity to aerosols by considering the variability across a set of measurements that would be made in a particular observational campaign. If we define $\sigma_{x}^{2}$ as the observed variance in parameter $x$ across this set of measurements, Equation 21.2 can be used to show that:

$$
\sigma_{c}^{2}=\lambda_{A_{\mu}}^{2} \sigma_{A_{\mu}}^{2}+\lambda_{M}^{2} \sigma_{M}^{2}+2 \lambda_{A_{\mu}} \lambda_{M} \sigma_{A_{\mu}} \sigma_{M} r
$$

where $r$ is the correlation coefficient between the $A_{\mu}$ and $M$. Equation $21.3 \mathrm{dem}-$ onstrates that not only do we need to understand the meteorological variability and its impact on the clouds, we also need to understand to what extent the meteorological variability covaries with the aerosol variability. In other words, accounting for the meteorological variability in a dataset (i.e., the second term in Equation 21.3, through knowledge of $\lambda_{M}$ ) is not sufficient to determine fully the aerosol sensitivity, if one does not also understand how the aerosol properties are tied to the large-scale flow. In addition, we note that such a formulation assumes a one-way cause-and-effect relationship, at least on short timescales, between aerosols and clouds and is a simplistic representation of a tightly coupled system in which clouds and aerosols interact on all scales. For instance, 
clouds can significantly impact aerosols via scavenging by precipitation, hence contributing to the covariance between aerosol and the meteorology. These mutual interactions raise serious concerns about the numerous and contrasting satellite studies that claim correlations between aerosols and clouds, which are generally interpreted as evidence of aerosols impacting clouds. In fact, quite the opposite may actually be true.

To optimize the chances of distinguishing an aerosol signal from the background meteorological noise, we must first select a place where the aerosol variability is significant, while the variability of the meteorology is minimized. The covariance between the two, however, sets a limit to this strategy: reducing meteorological variability (e.g., by selecting specific weather situations) necessarily reduces the aerosol variability. The second aerosol characterization experiment (ACE-2), which took place in the North Atlantic in June 1997, illustrates this impediment. Within ACE-2, the cloudy column experiment was designed to test the Twomey hypothesis; namely, that the cloud optical thickness, $\tau$, scales with the LWP, $W$, and the droplet number concentration $N$ as $\tau \propto W^{5 / 6} N^{1 / 3}$. The Atlantic Ocean, north of the Canary Islands, offers opportunities to sample air masses flowing around the Azores high that are generally pristine, except when they skim along the European continent (where they become polluted by anthropogenic aerosols). Droplet concentrations observed during the eight case studies ranged from less than $50 \mathrm{~cm}^{-3}$ in pristine air masses up to more than $250 \mathrm{~cm}^{-3}$ in the most polluted ones (i.e., a factor of five), whereas the LWP ranged from $33 \mathrm{~g} \mathrm{~m}^{-2}$ to $77 \mathrm{~g} \mathrm{~m}^{-2}$ (i.e., a factor of only two). Such a gap between aerosol and LWP variability allowed a precise validation of the Twomey hypothesis (discussed further below), but the dataset did not provide any evidence of an aerosol impact on the cloud life cycle. Indeed, aerosol and meteorology were closely correlated because polluted air masses had flown over the continent, and had hence experienced greater sensible and lower latent heat fluxes than the pristine oceanic air masses. Overall, the most polluted cases exhibited lower LWP than their pristine counterpart (Brenguier et al. 2003), and it was not feasible to determine whether clouds were thinner as a result of the reduced latent heat fluxes two days ahead over the continent, attributable to direct aerosol effects on the air mass when it moved over the ocean from the continent to the sampling area, or because of an indirect aerosol effect on the cloud layer.

Attempts were made to examine the climatology of precipitation downwind of large cities such as St. Louis, Missouri (Changnon et al. 1971). Careful analysis of the observations with a detailed numerical model indicated, however, that urban land use forced convergence downwind of the city, rather than the presence of greater aerosol concentrations, was the dominant control on the locations and amounts of precipitation in the vicinity of an urban complex (van den Heever and Cotton 2007).

An attractive alternative approach is to select situations where aerosol and meteorological variations are uncorrelated. This has served as the basis of 
weather modification control experiments for many years. Accidental biomass burning events offer such opportunities, but they are generally not frequent enough to build significant statistics. To improve the statistics, weekend effects have been examined climatologically (Forster and Solomon 2003; Gong et al. 2006). Evidence of a detectable weekly cycle of the diurnal temperature range has been shown, as well as the anthropogenic origin of this cycle. A potential aerosol effect on clouds was investigated by Bell et al. (2008), but may be masked by other atmospheric parameter changes (e.g., heat island effect) that are also correlated to the weekly cycle of anthropogenic activities. The identification of situations where aerosol variability is uncorrelated with meteorology thus remains to be resolved.

For shallow clouds, the issue of sensitivity is particularly acute. Indeed, their liquid water content is typically a few hundredths of the total water content. This explains why deriving their LWP and its temporal evolution from field observations of the thermodynamic fields (i.e. temperature and water vapor) remains beyond our grasp. The magnitude of the energy fluxes that govern the evolution of a cloud-topped boundary layer (e.g., surface fluxes including precipitation, cloud-top entrainment, and the flux divergences of short- and longwave radiation, $\lambda_{M}$ ) are comparable in magnitude to their potential modulation by the aerosol, for example by suppressing precipitation $\left(\lambda_{A \mu}\right)$. The susceptibility of marine stratocumulus clouds to aerosols is thus noticeable, as demonstrated by ship tracks.

Quantifying the susceptibilities of marine stratocumulus clouds to the meteorology and aerosol, respectively, is a challenge in the sense that small perturbations of the boundary layer state parameters need to be precisely measured. What facilitates the observation of these clouds, however, is their long synoptic lifetime (although, of course, the cloud element lifetime is only a few minutes), large spatial extension, relative statistical homogeneity at the mesoscale, and their reproducible diurnal cycle.

Beyond the susceptibilities, additional ingredients must also be carefully evaluated when designing a field experiment. This includes the spatial and temporal scales of interest, and the identification of all physical processes which may interfere with the observations. Next we describe two basic categories of observational approach that are particularly useful for the study of the interactions between marine stratocumulus clouds and their environment. The principles involved, however, are applicable to other cloud systems.

\section{Eulerian versus Lagrangian}

\section{Closure Experiments}

Closure experiments aim to measure the consistency of the atmospheric state parameters with respect to models of the underlying physical processes (Ogren 
1995; Quinn et al. 1996). The methodology consists of measuring input parameters to initialize a model and derive output parameters; concomitantly, the control parameters are measured for comparison with model predictions. To illustrate this, we will describe the observational strategy of the ACE-2 Cloudy-Column experiment (Brenguier, Chuang et al. 2000), which was the first field study dedicated entirely to the aerosol indirect effects in extended boundary-layer cloud systems. The hypotheses to be tested can be summarized in the form of three key questions:

1. For specified cloud fields, is the droplet concentration consistent with the predictions of aerosol activation models?

2. Do cloud radiative properties vary with droplet concentration, as anticipated by Twomey (1977)?

3. For a particular value of LWP, is the precipitation rate modulated by the droplet concentration?

\section{Temporal and Spatial Scales}

Sampling a single convective cell during its vertical ascent while measuring aerosol properties, vertical velocity, and cloud droplet concentration to examine $\mathrm{CCN}$ activation is not feasible using the existing airborne platforms. In addition, radiative transfer raises serious difficulties for single cells because it is, in essence, three dimensional and thus measurements of irradiance performed from above a single convective cell will necessarily be affected by radiation from neighboring cells. Finally, the cycle of precipitation formation in a single convective cell is short (a few tens of minutes), resulting in very heterogeneous drizzle patches below cloud base.

An alternative strategy is to examine the phenomenon at a larger scale at which aerosol properties, turbulence, cloud microphysics, and precipitation are statistically homogeneous (ergodic), and the three-dimensional heterogeneities of the radiation and precipitation fields are smoothed over a large number of cells. Such conditions are often satisfied in boundary-layer marine stratocumulus clouds at a scale of a few tens of kilometers.

\section{Aerosol Activation Closure}

This experiment aims to evaluate 0-D kinetic models of $\mathrm{CCN}$ activation to predict the cloud droplet concentration (control parameter) from the vertical velocity at cloud base and the physicochemical properties of the aerosol (input parameters) (Guibert et al. 2003; Snider et al. 2003).

Since we are unable to perform a closure experiment on individual CCN activation events, a statistical approach to the problem must be adopted, which necessarily must encompass the spatial variability of the system being studied. Aerosol properties can, far from the aerosol sources, be reasonably assumed to 
be uniform over the area and the duration of the experiment. Vertical velocity, on the other hand, varies from a few $\mathrm{cm} \mathrm{s}^{-1}$ up to more than $1 \mathrm{~m} \mathrm{~s}^{-1}$ in the most active cells. Thus, comparison involves the probability distribution function (PDF) of measured droplet concentration and its comparison with the predictions of a CCN activation model initialized with the full spectrum of measured vertical velocities. Figure 21.1 shows the comparison of the deciles of the measured droplet concentration PDF with the predictions of the model initialized successively with the deciles of the measured vertical velocity distribution. This figure demonstrates that the range of concentration variability resulting from vertical velocity fluctuations is broader than the difference between the mean values of a pristine and a polluted case.

Therefore, a closure experiment on $\mathrm{CCN}$ activation will not be conclusive if the vertical velocity is not fully constrained by observations. A consistent definition of the cloud droplet concentration used here as a control parameter is also crucial. In fact, the droplet concentration measured in a cloud system is different from the one resulting from $\mathrm{CCN}$ activation, even though both are tightly related. After $\mathrm{CCN}$ activation is completed, additional processes (e.g., mixing with the environmental dry air and scavenging by precipitation) dilute droplet concentration significantly. Thus, for comparison with a CCN activation model prediction, it is sensible to select only those droplet concentration samples that are not affected by either mixing or precipitation scavenging. In ACE-2, for instance, the droplet concentration after selection was

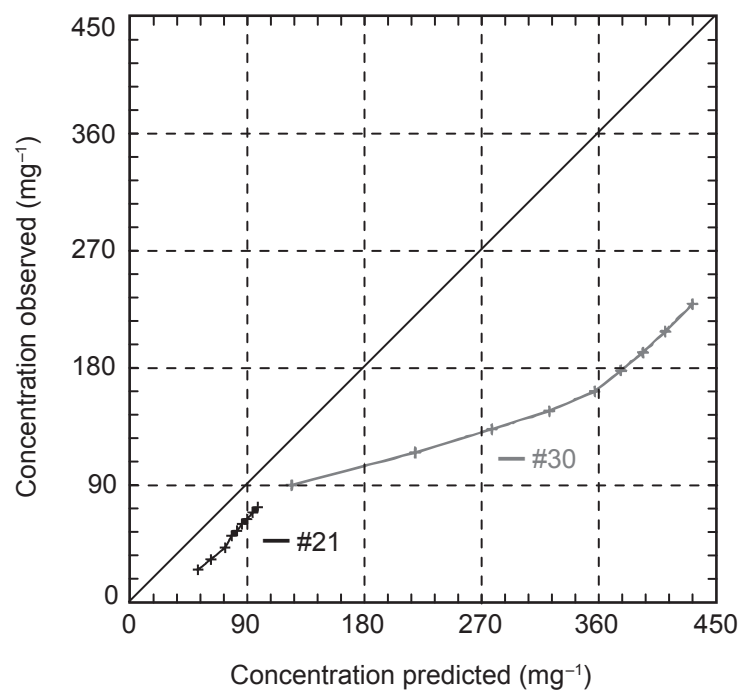

Figure 21.1 Deciles of the observed droplet concentration probability distribution function (PDF) versus predictions of the droplet concentration with a CCN activation model initialized successively with the deciles of the observed vertical velocity PDF for flight 21 (pristine case) and flight 30 (polluted case). 
typically 30\% higher than the average over all the samples (Pawlowska and Brenguier 2000).

The main limitation of this closure experiment was the incomplete characterization of the size-segregated chemical composition of the particles, which introduces uncertainties in the prediction of their hygroscopic properties. Since the development of airborne mass spectrometers and improved chemical analysis, activation closure has been significantly refined (Conant et al. 2004; Fountoukis et al. 2007). Further work remains to be conducted to characterize the mass accommodation coefficient for small growing particles, and instruments need to be designed to characterize their state of chemical mixture.

\section{Column Closure on Radiative Transfer}

This type of experiment aims to corroborate the Twomey hypothesis (i.e., that aerosol-induced microphysical changes are reflected by changes of cloud radiative properties). The input parameters measured in situ are the vertical distribution and horizontal variability of the cloud droplet size distribution, and the output parameter is the optical thickness of the cloud layer, derived as the vertical integral of extinction. The control parameter is the optical thickness derived independently from multispectral radiances measured with a second aircraft flying above the cloud layer.

In ACE-2, the statistics of all input parameters were very robust because of the long duration of sampling with two collocated aircraft: one was dedicated to the microphysical fields while the other focused on radiation. Thus, ACE-2 provided the first observational evidence of the Twomey effect in extended cloud systems, e.g., the scaling of the optical thickness with LWP and droplet concentration as anticipated by Twomey (see Fig. 6 in Brenguier, Pawlowska et al. 2000). One serious limitation, however, was that in-situ airborne measurements do not provide information on how the microphysical fields sampled at various levels are distributed vertically. The optical thickness used as the output parameter was derived by assuming either random or maximum overlap of the microphysical fields measured in situ. The accuracy of the prediction was thus significantly degraded by the overlap uncertainty. Since ACE-2, other closure experiments have been performed using remote-sensing systems that better constrain the vertical organization of the microphysical fields (Feingold et al. 2003).

An alternative approach to radiative closure is to validate the same radiative transfer model but in the inverse mode. Indeed, inverse models are currently used to derive cloud properties from space measurements of multispectral radiances (Nakajima and King 1990). In this approach, radiance measurements are used to derive cloud geometrical thickness, or LWP, and droplet concentration, which are then compared to the ones measured in situ (Schüller et al. 2003). 


\section{Column Closure on Precipitation}

This experiment contributes to the improvement of precipitation formation parameterization in global climate models. Recent field studies suggest that the precipitation rate of stratocumulus clouds, averaged over a large domain containing numerous cloud cells, scales with the mean cloud thickness or LWP and the typical droplet concentration: ACE-2 (Pawlowska and Brenguier 2003), EPIC (Comstock et al. 2004; Wood 2005), and DYCOMS-II (Van Zanten et al. 2005). The nature of such a relationship is a major determinant of the magnitude of the aerosol impact on cloud extent thickness and lifetime. Large eddy simulations (LES) are therefore used to corroborate these observations and quantify the empirical relationship better.

The observations are summarized in Figure 21.2a. For each field campaign, the precipitation rate at cloud base scales well with the cloud thickness and cloud droplet concentration. Each dataset appears, however, to have offsets that mainly reflect measurement biases and differences in the methodology: precipitation rate averaged over the cloud layer (ACE-2) or at cloud base only (EPIC and DYCOMS-2), droplet concentration measured in cloud samples that are not affected by mixing or precipitation scavenging (ACE-2), averaged over the cloud layer (DYCOMS-II), or extrapolated from remote sensing (EPIC), cloud thickness derived from detection of cloud base and top (ACE-2 and DYCOMS-II) or derived from remote sensing of the LWP (EPIC). These discrepancies reveal how sensitive the results can be to the definition of the physical parameters derived from diverse measurement and data processing techniques. In addition, the results demonstrate just how sensitive the precipitation rate is to cloud macrophysical properties, with a doubling of precipitation requiring only a change of $\sim 100 \mathrm{~m}$ in cloud thickness. This emphasizes the importance of controlling for meteorological variability when examining microphysical impacts.

Numerical simulations of similar cloud systems were performed with an LES model over a broad range of LWP and CCN concentration values to explore the parameter space of the measurements (Geoffroy et al. 2008). Figure $21.2 \mathrm{~b}-\mathrm{d}$ show the comparison of the model results with the measurements, using the same parameters and scaling laws as in each field campaign, respectively. The similarity between observations from three different areas and the results of numerical simulation suggests that the large-scale relationship between LWP, droplet concentration, and the precipitation rate at cloud base is robust.

\section{Summary and Recommendations}

These three examples illustrate different types of closure experiments. Each type has clearly testable hypotheses. When the model is straightforward, such as the 0 -D model of CCN activation, the closure experiment follows closely 
the basic methodology: measured input parameters, numerical simulations, comparison of model predictions with the control parameter. In the second example, the distinction between input and control parameters is less obvious, depending on whether the model is used in the direct or in the inverse mode, such as for satellite 1-D retrieval techniques. The third example, with its 3-D model of stratocumulus clouds, suggests how the technique can be extrapolated to compare relationships between specified physical parameters that have been observed and further simulated with a model over the same parameter space. The three approaches share in common the following methodological rules, which are generally not given sufficient attention in most of the closure studies:

1. Models need to be fully constrained: All parameters, which might impact the prediction of the model to be tested, must be documented according to a level of accuracy consistent with their impact (e.g., as for vertical velocity).

2. Ensure consistency in the definition of the measured and model parameters: The measured and model values of a parameter must be defined over the same spatial and temporal scales. For example, droplet concentration will exhibit significant differences, depending upon whether it is defined as the mean value over a cloud system or the value specifically measured in regions of CCN activation.

3. Redundancy in measurements is highly desirable: Single validation experiments often succeed, whereas redundant controls are more difficult to reconcile, but allow for a higher degree of confidence. For closure to be robust, attempts must be made to combine redundant closures of the same process, such as combining a CCN activation spectrum and a droplet activation closure on the same data set (Snider et al. 2003) or radiation closures on both transmitted and reflected light in the same cloud system (Platnick 2000).

In general, dynamic, thermodynamic, and microphysical properties exhibit important variability and covariability on the kilometer scale (i.e., scales smaller than a typical climate model grid box), and this variability has marked impacts on how aerosol-cloud interaction affects the large-scale properties of clouds. It is important to design a sampling strategy that allows us to characterize further these important subgrid statistical connections between variables (see, e.g., Illingworth and Bony, this volume; Larson et al. 2001, 2002). For instance, airborne measurements in clouds are often optimized by targeting cloud cells along the flight track; however, such an approach introduces bias into the data base (overestimated cloud fraction). Thus, it is crucial to adopt unbiased sampling or provide additional information to reduce potential biases in the data base.

Column closure experiments are useful to validate models of physical processes and their parameterizations for general circulation models, as long as the 
(a)

(b)
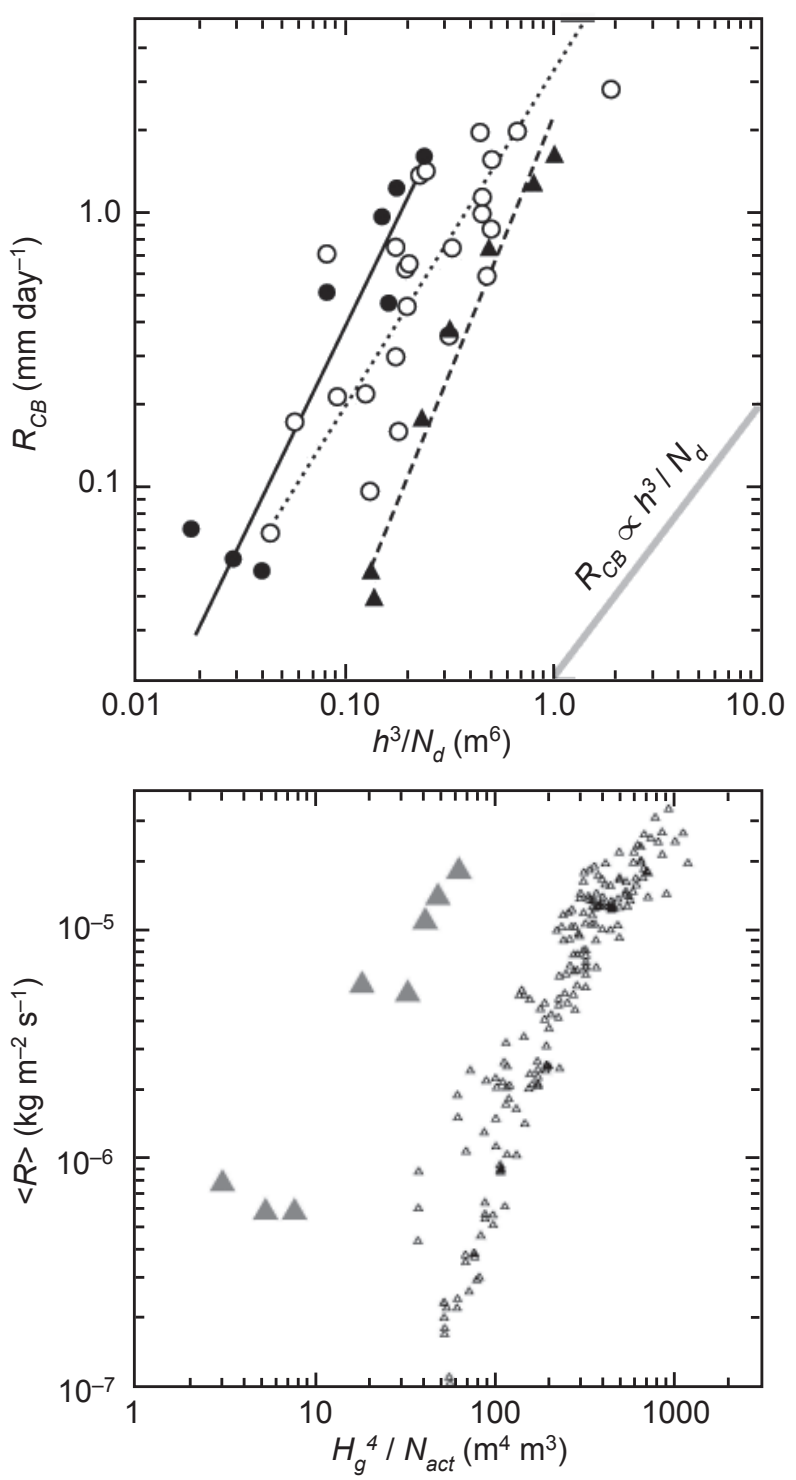

Figure 21.2 (a) Cloud base precipitation rates, $R_{C B}$, from observational case studies in subtropical marine stratocumulus, plotted against $h^{3} / N_{d}$, where $h$ is the cloud thickness and $N_{d}$ the droplet concentration. Black circles: Pawlowska and Brenguier (2003), in-situ aircraft; white circles: Comstock et al. (2004), radiometric and radar drizzle; triangles: van Zanten et al. (2005), in-situ aircraft and radar drizzle. The lines represent linear least-distance regressions to the case studies for each field campaign. Comparison of model predictions (small triangles) with scaling laws derived from: (b) ACE-2 (large triangles): precipitation rate $<\mathrm{R}>$ averaged over the cloud layer, cloud thickness $H_{g}$ 
(c)

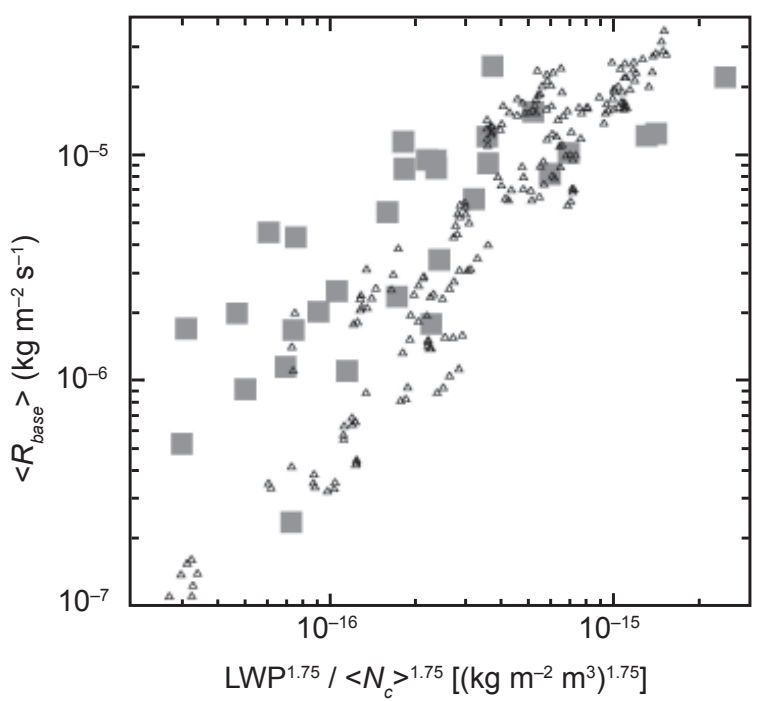

(d)

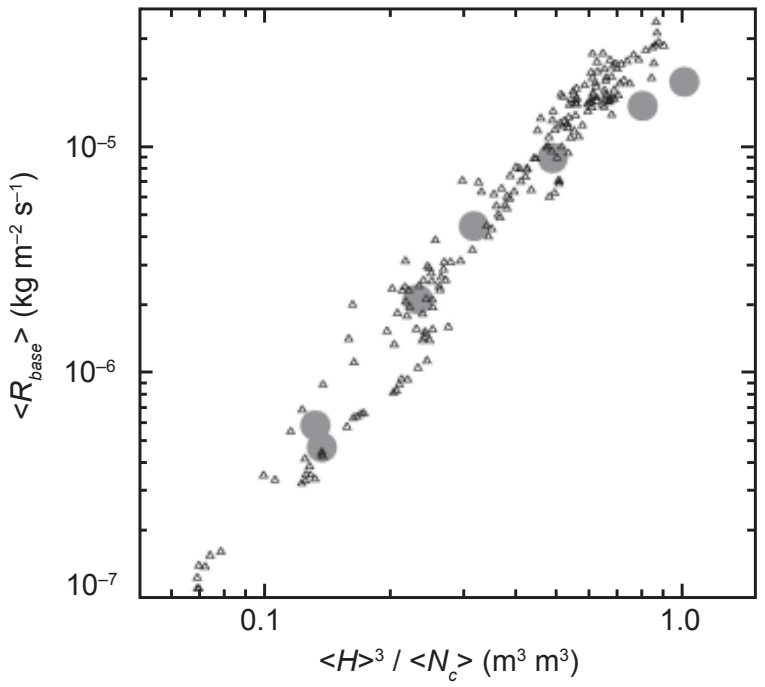

Figure 21.2 (continued) derived from in-situ measurements and droplet concentration, $N_{a c t}$, derived from samples not affected by mixing or droplet scavenging. (c) EPIC (squares): precipitation rate at cloud base $\left\langle R_{\text {base }}>\right.$, liquid water path (LWP), and mean droplet concentration $N_{c}$, derived from surface remote sensing. (d) DYCOMS-II) (circles): precipitation rate at cloud base $\left\langle R_{\text {base }}>\right.$ and cloud thickness $\langle H\rangle$ derived from remote sensing and mean droplet concentration, $N_{c}$, derived from in-situ measurements. 
system is evolving sufficiently slowly. They are not suited, however, for studies of the temporal evolution of the system, which call for a different approach.

\section{Lagrangian Experiments: Linking Small and Large Scales}

\section{The Lagrangian Concept}

The temporal and spatial scales over which cloud fields evolve and interact with the general circulation in which they are embedded are typically days and thousands of kilometers. Scales involved in the general circulation exceed those accessible to single aircraft flights and limit seriously our ability to understand the two-way interactions between microscale processes and the general circulation.

This need to observe the evolution of meteorological fields and the processes that influence their development over long timescales led to the development of the Lagrangian observational concept, which seeks to make quasicontinuous observations of an air mass (ideally over several days, although as we shall see there are considerable benefits from flights lasting only a few hours) often by employing multiple observation platforms which overlap in time. The Lagrangian observational approach aims to study processes within

a frame of reference that moves with the air, an approach necessary to study time-evolving processes in the atmosphere without resorting to making assumptions about poorly constrained advective processes.

Despite the high costs associated with fully realizing the potential of the Lagrangian approach using in-situ aircraft observations, a number of such observational studies have been conducted over the last few decades. These studies have yielded a tremendously rich understanding of a wide variety of different processes, as we discuss below.

\section{Lagrangian Boundary Layer and Cloud Evolution}

Lagrangian sampling of cloud-related processes on timescales of a day or more has primarily focused upon the marine boundary layer (MBL), because many of the key processes relevant to climate occur there. In addition, the logistics associated with conducting a Lagrangian study in the lowest level are simpler (not least of which is the presence of a solid and uniform boundary at the base of the air mass). Although earlier Lagrangian studies involved balloons and tracers, the first multi-flight Lagrangian experiments devoted to understanding cloud processes were conducted during the Atlantic Stratocumulus Transition Experiment/Marine Aerosol and Gas Experiment (ASTEX/MAGE) in June 1992 (Albrecht et al. 1995; Businger et al. 2006). Two such studies were carried out, each for a period of 36-48 hours ( $~ 800 \mathrm{~km}$ of spatial advection). During these studies, constant-density balloons floated downwind with the air mass and radioed their GPS-derived locations to a relay of three sampling 
aircraft. The balloons served as markers for the boundary-layer air mass, which was continually being modified by chemical and energy fluxes at the surface, entrainment of free tropospheric air, wind shear within the boundary layer, horizontal dispersion, chemical reactions, and aerosol transformations. Here we will focus on those aspects most pertinent to clouds.

The ASTEX/MAGE Lagrangian experiments were revolutionary for a number of reasons. By continuously sampling the MBL over a period approaching two days, it was possible to make observations of the transition from a shallow, well-mixed stratocumulus-capped MBL to a much deeper, decoupled MBL containing trade cumuli of less extensive coverage (Bretherton and Pincus 1995). Such air mass transitions are a critical component of the transition from the subtropical to the tropical MBL, and the physical mechanism by which the MBL undergoes this transition was deduced directly as a result of the Lagrangian observations. Previous hypotheses for the transition followed ideas based upon the work on diurnally modulated decoupling of the boundary layer that had been carried out at fixed locations. The ASTEX Lagrangian data were able to demonstrate that although decoupling of the MBL is a key process in the transition, it is actually the increasing latent heat flux as the air mass moves over warmer water, rather than solar radiation, that is the key driver of the subtropical transition (Bretherton and Wyant 1997).

\section{Lagrangian Entrainment Rate Estimates}

A second novel aspect of Lagrangian studies is the ability to derive direct estimates of the rate of MBL deepening, and thus the entrainment rate. It is now widely appreciated just how important the entrainment rate is, not only for its physical impacts upon the MBL thermodynamic and cloud climatology (Stevens 2002), but also because entrainment is a critical mediator of the response of cloud field to perturbations in atmospheric aerosols (Ackerman et al. 2004). Because microphysically induced changes (e.g., suppression of drizzle) impact the entrainment rate by changing the MBL turbulent structure, this introduces much longer timescales than the Twomey effect (Wood 2007), which cannot be quantified observationally using measurements from fixed locations.

ASTEX allowed Lagrangian estimates of the entrainment rate over the two days of observations (Bretherton et al. 1995). Recent studies have employed the Lagrangian technique using flight durations of only a few hours. For example, in DYCOMS-II (Stevens et al. 2003), single 6-hr Lagrangian flights using a C-130 aircraft were used to ascertain the entrainment rate by determining the observed rate of boundary layer deepening and subtracting the subsidence rate from reanalysis data (Faloona et al. 2005). This was then compared with the entrainment rate estimated using the standard flux-jump method to provide closure on the time evolution of the entrainment rate that can be used to constrain model ability to represent the entrainment process correctly. 


\section{Aerosol-Cloud-Chemistry Interactions using Lagrangians}

Although aerosol indirect effects were not a specific focus of the ASTEX Lagrangians, the aerosol and gas phase chemistry measurements taken as part of the MAGE component of the campaign provided the first concrete demonstration of the benefits that the Lagrangian observational concept could bring to our understanding of chemical process rates in the atmosphere (Zhuang and Huebert 1996; Clarke et al. 1996), a need that had been identified since the early 1970s. One might argue that the development of the sampling concept for the early Lagrangian studies led to the realization that aerosol particles and their precursors are themselves a fundamental component of the atmospheric general circulation, which thereby provides further possibilities for interactions between these scales that were not previously appreciated.

Lagrangian experiments that focused specifically upon aerosols and aerosol precursors were conducted as part of the First and Second Aerosol Characterization Experiments (ACE-1, ACE-2) in 1995 and 1997, respectively (Bates et al. 1998; Johnson et al. 2000). They provided a wealth of data on the coupling between aerosols, aerosol precursors, clouds, and the MBL, in both pristine and polluted MBLs. From the ACE-2 Lagrangian studies it has been possible to assemble a conceptual model for continental pollution outbreaks that serves as an invaluable basis for the planning of future sampling of the modified continental plume. Timescale analysis applied to the ACE-2 Lagrangian datasets (Hoell et al. 2000) demonstrated the importance of meteorological factors (e.g., dilution) in controlling the properties of aerosols in the MBL. The example from the third Lagrangian experiment during ACE2, shown in Figure 21.3, demonstrates the ability of Lagrangian experiments to connect micro- and mesoscale characteristics with those of the large-scale flow.

\section{A Strategy for Future Lagrangian Experiments}

Although they have provided invaluable information about air mass transformations and the physical and chemical processes within them, the Lagrangian experiments performed to date have not yet reached their full potential as important strategies for determining how clouds will change in a perturbed climate:

1. Sampling limitations: To date, Lagrangian studies have sampled with one aircraft at any one given time, whereas current state-of-the-art observational techniques require the use of multiple aircraft (e.g., column closure experiments discussed above) to link the aerosol, thermodynamic, and dynamic properties of the atmosphere measured in situ with the column cloud radiative properties remotely sensed from above. Adopting this strategy within a Lagrangian experiment would be very powerful, especially if scanning radiometers could be used to 


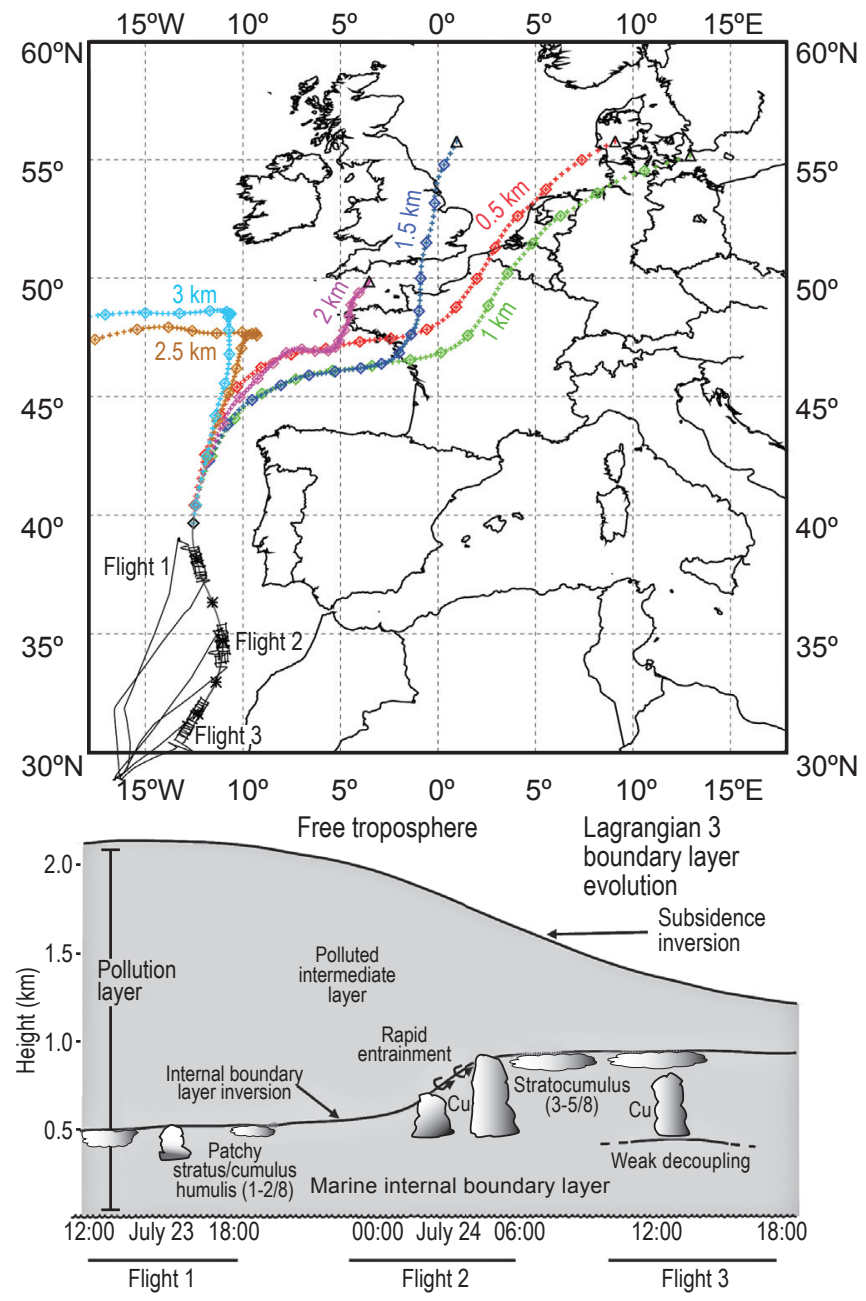

Figure 21.3 Flight tracks (top panel) for the third Lagrangian experiment of ACE-2 (Wood et al. 2000) which sampled a polluted continental outbreak heading southward over the northeast subtropical Atlantic Ocean. Back trajectories, estimated using ECMWF analyses, are shown ending at different altitudes at the location of the start of the sampling. The three flights allow us to construct a picture of the changing structure of the lowest $2 \mathrm{~km}$ of the atmosphere, the essence of which is detailed schematically (bottom panel). Modified from Wood et al. (2000).

broaden the measurements to be representative of an area. Moreover nadir-pointing remote-sensing systems provide crucial information on how microphysical fields that are sampled horizontally by in-situ aircraft overlap vertically (Damiani et al. 2006).

2. New sampling techniques: Beyond piloted aircraft, new vectors are becoming available for in-situ sampling of boundary-layer clouds at 
low speed. Unmanned aerial vehicles have a typical sampling speed of $30 \mathrm{~m} \mathrm{~s}^{-1}$ and they can now fly autonomously a predefined track (Ramanathan et al. 2007). Blimps have been used in the past and new platforms (e.g., the Zeppelin) might be used for Lagrangian studies, although the logistics involved remain a serious obstacle.

3. Interdisciplinary spirit: To characterize the evolution of the cloud layer fully, it is important to have a detailed characterization of all the potential factors controlling it, including the large-scale meteorology, the surface and MBL top boundary conditions (both physical and chemical) as well as the cloud microphysical, thermodynamic, and turbulent information. This is a tall order for a single platform but can be achieved, provided there is effective interdisciplinary communication and adequate financial support. The forthcoming EUCAARI and VOCALS experiments provide good examples of the possible connections that can be made between research communities (oceanography, atmospheric chemistry, cloud physics, and climate dynamics).

4. Synergy between models and observation: The most serious obstacle in the characterization of a boundary layer is our inability to measure the thermodynamic conservative variables (total heat and moisture) and their budgets accurately enough to constrain the resulting LWP. However, LES models, which can simulate the evolution of a boundary layer, suggest observable signatures of the mechanisms that drive its evolution. For instance, Sandu et al. (2008) showed that the impacts of the aerosol on the diurnal cycle of stratocumulus clouds are reflected by observable differences in the vertical velocity variance at cloud top, in the vertical profile of liquid water content, and in the level of turbulent kinetic energy below cloud base.

5. Complementary information: With the current generation of satellite remote sensing, there is a tremendous amount of additional information that can be added from space to multi-day Lagrangian studies. Geostationary satellites, especially those with high-resolution $(1 \mathrm{~km})$, multi-channel and high-sampling frequency (15 min for SEVIRI on MSG) spectrometers can provide cloud microphysical, aerosol, and cloud-top temperature information. Polar-orbiting satellites with active remote sensing, such as in the A-Train, provide additional information, at least once a day, to calibrate geostationary satellite observations better. Promising techniques appear to derive accurate temperature, humidity, and boundary-layer depth information from spaceborne GPS limb sounding. A reanalysis focused on clouds, aerosols, and the boundary layer will be an excellent way to incorporate the satellite information into a useable framework. High-resolution numerical modeling could be calibrated using in-situ data to "fill in the gaps" in rather the same way that assimilation is used in the numerical weather prediction community. 
In addition, aircraft observations do not provide complete information about the large-scale dynamic fields; key variables, such as the large-scale vertical motion field, are completely unknown. These fields are critically important for understanding the interactions between the small and large scales and for allowing a top-down assessment of the entrainment rate that can be compared with bottom-up measurements carried out by integrating over many turbulent eddies. This is currently accomplished by using standard reanalysis products, but these are not generally produced with the needs of the cloud-climate community in mind.

\section{General Recommendations and Conclusions}

Understanding how clouds respond to a changing climate requires carefully designed micro- and mesoscale observational experiments. The task of separating the impacts of variability in large-scale meteorology from those caused by changes in aerosol physical and chemical properties is very challenging. This is because cloud and precipitation formation is sensitive to both changes in the large-scale forcings as well as to microphysical aerosol-induced impacts. Moreover, not only the instantaneous relationships between microphysical and macrophysical variables, but also the temporal evolution of the fields must be documented. Thus, it is important to put the observed microphysical changes in perspective with the effects of the large-scale meteorological variability.

\section{Need for Better Connection between Small and Large Scales}

Acquiring a better understanding of the interactions between meso- and microscale processes and the large-scale flow requires the provision of accurate boundary conditions for meso- and microscale studies. In recent years, this has been achieved using large-scale meteorological data from reanalysis datasets (e.g., Kistler et al. 2001; Uppala et al. 2005), which have proven extremely useful because they provide gridded data at regular temporal intervals everywhere in the atmosphere.

Current reanalyses place a high value on creating datasets that are homogeneous over a long period of time (40 years), hence preventing the use of the most advanced, but computationally expensive, models as well as limiting assimilation to data that are available over the whole reanalysis period, or at least relatively lengthy fractions of it. Aerosol processes, for instance, are not represented at all in current reanalyses; however, given the recent advances to our observing system, this should be possible. We argue that the "one-sizefits-all" approach is not optimal to address the cloud-climate problem. Indeed, we believe that focused reanalysis systems, which target key processes and assimilate new datasets, would be of major benefit to the observational community. These systems would not necessarily be global, but could instead be 
run over much smaller spatial and temporal domains at higher resolution. Regional reanalysis (e.g., the North American regional reanalysis) is a move in this direction. Incorporating physical process specialization with geographical specialization would also be a beneficial development. An assimilation system that focuses upon the lower troposphere, including the boundary layer, would greatly benefit our understanding of low cloud systems and their relationship with large-scale meteorological and aerosol forcings.

To optimize boundary conditions, the instrumental setup should be designed to connect the meso- to the microscale. This should rely on a combination of large-scale, though limited and discontinuous, sampling with instrumented aircraft and surface (either ground or ship) stations that provide continuous sampling of the atmosphere flowing above the site.

\section{Need for Consistent Multidisciplinary Approaches}

Clouds are perturbed in their dynamics, microphysics, and radiative properties. Field experiments dedicated to this challenging issue must be designed with an instrumental setup that permits the documentation of each source of perturbation and each effect with accuracies consistent with their anticipated contribution or level of response to the forcings. New airborne instruments are now available to document crucial parameters, such as the size-segregated chemical composition of the aerosol. Considerable progress has been made to overcome the sampling limitation of instrumented aircraft by using airborne active remote-sensing systems that provide crucial information about the vertical organization of the cloud systems sampled horizontally by the aircraft (e.g., Damiani et al. 2006). We argue that instead of running numerous separate partial studies that do not consider the critical atmospheric parameters well enough, efforts should be concentrated on large-scale multidisciplinary and multiplatform projects.

\section{Detailed Planning}

Most surface remote-sensing systems sample only two-dimensional slices of atmosphere, and instrumented aircraft samples are limited to long lines of data of a few $\mathrm{mm}^{2}$ cross section each. Their use, over the short duration of an intensive observation period, must therefore be highly optimized; compromises must be made on where to locate surface systems and which flight tracks to allocate to each aircraft. Thus, the preparation of a field experiment involves preliminary studies of the expected phenomena to identify very specific and observable signatures of the physical processes and mechanisms that are supposed to express the impact of the large-scale meteorological and microscale aerosol forcings. The use of large-scale modeling, process modeling, and theory is extremely helpful in designing testable hypotheses which observational datasets can help 
to refute. A clear and precise definition of the sampling strategy is by far the most important ingredient of a micro- and mesoscale observation program.

\section{Acknowledgments}

We thank Bruce Wielicki for discussions from which the ideas for an improved reanalysis focused upon the boundary layer emerged.

\section{References}

Ackerman, A. S., M. P. Kirkpatrick, D. E. Stevens, and O. B. Toon. 2004. The impact of humidity above stratiform clouds on indirect aerosol climate forcing. Nature 432:1014-1017.

Albrecht, B. A., C. S. Bretherton, D. Johnson, W. H. Schubert, and A. S. Frisch. 1995. The Atlantic stratocumulus experiment: ASTEX. Bull. Amer. Meteor. Soc. 76:889-904.

Bates, T. S., B. J. Huebert, J. L. Gras, F. B. Griffiths, and P. A. Durkee. 1998. International Global Atmospheric Chemistry (IGAC) project's first Aerosol Characterization Experiment (ACE 1): Overview. J. Geophys. Res. 103:16,297-16,318.

Bell, T. L., D. Rosenfeld, K.-M. Kim et al. 2008. Midweek increase in U.S. summer rain and storm heights suggests air pollution invigorates rainstorms. J. Geophys. Res. 113:D02209.

Brenguier, J. L., P. Y. Chuang, Y. Fouquart et al. 2000. An overview of the ACE-2 CLOUDYCOLUMN closure experiment. Tellus 52B:814-826.

Brenguier, J. L., H. Pawlowska, and L. Schüller. 2003. Cloud microphysical and radiative properties for parameterization and satellite monitoring of the indirect effect of aerosol on climate, PACE Topical Issue. J. Geophys. Res. 108(D15):8632.

Brenguier, J. L., H. Pawlowska, L. Schueller et al. 2000. Radiative properties of boundary layer clouds: Droplet effective radius versus number concentration. $J$. Atmos. Sci. 57:803-821.

Bretherton, C. S., P. Austin, and S. T. Siems. 1995. Cloudiness and marine boundary layer dynamics in the ASTEX Lagrangian experiments. Part II: Cloudiness, drizzle, surface fluxes and entrainment. J. Atmos. Sci. 52:2724-2735.

Bretherton, C. S., and R. Pincus. 1995. Cloudiness and marine boundary layer dynamics in the ASTEX Lagrangian experiments. Part I: Synoptic setting and vertical structure. J. Atmos. Sci. 52:2707-2723.

Bretherton, C. S., and M. C. Wyant. 1997. Moisture transport, lower-tropospheric stability, and decoupling of cloud-topped boundary layers. J. Atmos. Sci. 54:148167.

Businger, S., R. Johnson, and R. Talbot. 2006. Scientific insights from four generations of Lagrangian smart balloons in atmospheric research. Bull. Amer. Meteor. Soc. 87:1539-1554.

Changnon, S. A., F. A. Huff, and R. G. Semonin et al. 1971. An investigation of inadvertent weather modification. Bull. Amer. Meteor. Soc. 52:958-968.

Clarke, A. D., T. Uehara, and J. N. Porter. 1996. Lagrangian evolution of an aerosol column during the Atlantic Stratocumulus Transition Experiment. J. Geophys. Res. 101:4351-4362. 
Comstock, K. K., R. Wood, S. E. Yuter, and C. S. Bretherton. 2004. Reflectivity and rain rate in and below drizzling stratocumulus. Q. J. Roy. Meteor. Soc. 130:2891-2919.

Conant, W. C., T. M. VanReken, T. A. Rissman et al. 2004. Aerosol-cloud drop concentration closure in warm cumulus. J. Geophys. Res. 109:D13204.

Damiani, R., G. Vali, and S. Haimov. 2006. The structure of thermals in cumulus from airborne dual-doppler radar observations. J. Atmos. Sci. 63:1432-1450.

Faloona, I., D. H. Lenschow, T. Campos et al. 2005. Observations of entrainment in eastern Pacific marine stratocumulus using three conserved scalars. J. Atmos. Sci. 62:3268-3285.

Feingold, G., L. E. Wynn, D. E. Veron, and M. Previdi. 2003. First measurements of Twomey indirect effect using ground-based remote sensors. Geophys. Res. Lett. 30:1287.

Forster, P., V. Ramaswamy, P. Artaxo, et al. 2007. Changes in atmospheric constituents and in radiative forcing. In: Climate Change 2007: The Physical Science Basis. Contribution of Working Group I to the Fourth Assessment Report of the Intergovernmental Panel on Climate Change, ed. S. Solomon, S. D. Qin, M. Manning et al., pp. 129-234. Cambridge: Cambridge Univ. Press.

Forster, P., and S. Solomon. 2003. Observations of a "weekend effect" in diurnal temperature range. PNAS 100:11,225-11,230.

Fountoukis, C., A. Nenes, N. Meskhidze et al. 2007. Aerosol-cloud drop concentration closure for clouds sampled during ICARTT. J. Geophys. Res. 112:D10S30.

Geoffroy, O., J. L. Brenguier, and I. Sandu. 2008. Relationship between drizzle rate, liquid water path and droplet concentration at the scale of a stratocumulus cloud system. Atmos. Chem. Phys. Discuss. 8:3921-3959.

Gong, D.-Y., D. Guo, and C.-H. Ho. 2006. Weekend effect in diurnal temperature range in China: Opposite signals between winter and summer. J. Geophys. Res. 111:D18113.

Guibert, S., J. R. Snider, and J. L. Brenguier. 2003. Aerosol activation in marine stratocumulus clouds part I: Measurement validation for a closure study PACE topical issue. J. Geophys. Res. 108(D15):8628.

Held, I. M., and B. J. Soden. 2006. Robust responses of the hydrological cycle to global warming. J. Climate 19(21):5686-5699.

Hoell, C., C. O'Dowd, S. Osborne, and D. Johnson. 2000. Timescale analysis of marine boundary layer aerosol evolution: Lagrangian case studies under clean and polluted cloudy conditions. Tellus 52B:423-438.

IPCC. 2007. Climate Change 2007: The Physical Science Basis. Contribution of Working Group I to the Fourth Assessment Report of the Intergovernmental Panel on Climate Change, ed. S. Solomon, D. Qin, M. Manning et al. New York: Cambridge Univ. Press.

Johnson, D. W., S. R. Osborne, R. Wood et al. 2000. An overview of the Lagrangian experiments undertaken during the second Aerosol Characterisation Experiment. Tellus 52B:290-320.

Kistler, R., E. Kalnay, W. Collins et al. 2001. The NCEP-NCAR 50-year reanalysis: Monthly means CD-ROM and documentation. Bull. Amer. Meteor. Soc. 82:247-267.

Klein, S. A., and D. L. Hartmann. 1993. The seasonal cycle of low stratiform clouds. J. Climate 6:1588-1606.

Klein, S. A., D. L. Hartmann, and J. R. Norris. 1995. On the relationships among low-cloud structure, sea surface temperature, and atmospheric circulation in the summertime northeast Pacific. J. Climate 8:1140-1155. 
Larson, V. E., J.-C. Golaz, and W. R. Cotton. 2002. Small-scale and mesoscale variability in cloudy boundary layers: Joint probability density functions. J. Atmos. Sci. 59:3519-3539.

Larson, V. E., R. Wood, P. R. Field, J.-C. Golaz, and T. H. Vonder Haar. 2001. Small-scale and mesoscale variability of scalars in cloudy boundary layers: One-dimensional probability density functions. J. Atmos. Sci. 58:1978-1994.

Lau, K. M., M. K. Kim, and K. M. Kim. 2006. Asian summer monsoon anomalies induced by aerosol direct forcing: The role of the Tibetan Plateau. Climate Dyn. 26:855-864.

Nakajima, T., and M. D. King. 1990. Determination of the optical thickness and effective radius of clouds from reflected solar radiation measurements, Part I: Theory. J. Atmos. Sci. 47:1878-1893.

Ogren, J. A. 1995. A systematic approach to in situ observations of aerosol properties. In: Aerosol Forcing of Climate, ed. R. J. Charlson and J. Heintzenberg, pp. 215-226. Chichester: Wiley.

Pawlowska, H., and J. L. Brenguier. 2000. Microphysical properties of stratocumulus clouds during ACE-2. Tellus 52B:867-886.

Pawlowska, H., and J. L. Brenguier. 2003. An observational study of drizzle formation in stratocumulus clouds during ACE-2 for GCM parameterizations. PACE Topical Issue, J. Geophys. Res. 108(D15):8630.

Platnick, S. 2000. Vertical photon transport in cloud remote sensing problems. J. Geophys. Res. 105:22,919-22,935.

Quinn, P. K., T .L. Anderson, T. S. Bates et al. 1996. Closure in tropospheric aerosolclimate research: A review and future needs for addressing aerosol direct shortwave radiative forcing. Contrib. Atmos. Phys. 69(4):547-577.

Ramanathan, V., M. Ramana, G. Roberts et al. 2007. Warming trends in Asia amplified by brown cloud solar absorption. Nature 448:575-578.

Randall, D. A., R. A. Wood, S. Bony et al. 2007. Climate models and their evaluation. In: Climate Change 2007: The Physical Science Basis. Contribution of Working Group I to the Fourth Assessment Report of the Intergovernmental Panel on Climate Change, ed. S. Solomon, S. D. Qin, M. Manning et al., pp. 589-662. Cambridge: Cambridge Univ. Press.

Sandu, I., J. L. Brenguier, O. Geoffroy, O. Thouron, and V. Masson. 2008. Aerosol impacts on the diurnal cycle of marine stratocumulus. J. Atmos. Sci., in press.

Schüller, L., J. L. Brenguier, and H. Pawlowska. 2003. Retrieval of microphysical, geometrical and radiative properties of marine stratocumulus from remote sensing, PACE topical issue. J. Geophys. Res. 108(D15):8631.

Snider, J. R., S. Guibert, and J. L. Brenguier. 2003. Aerosol activation in marine stratocumulus clouds, part II: Köhler and parcel theory closure studies, PACE topical issue. J. Geophys. Res. 108(D15):8629.

Stevens, B. 2002. Entrainment in stratocumulus topped mixed layers. Q. J. Roy. Meteor. Soc. 128:2663-2690.

Stevens, B., D. H. Lenschow, G. Vali et al. 2003. Dynamics and chemistry of marine stratocumulus, DYCOMS-II. Bull. Amer. Meteor. Soc. 84:579-593.

Twomey, S. A. 1977. The influence of pollution on the shortwave albedo of clouds. J. Atmos. Sci. 34:1149-1152.

Uppala, S. M., P. W. Kållberg, A. J. Simmons et al. 2005. The ERA-40 reanalysis. Q. J. Roy. Meteor. Soc. 131:2961-3012.

van den Heever, S., and W. R. Cotton. 2007. Urban aerosol impacts on downwind convective storms. J. Appl. Meteor. Climatol. 46:828-850. 
Van Zanten, M. C., B. Stevens, G. Vali, and D. Lenschow. 2005. Observations of drizzle in nocturnal marine stratocumulus. J. Atmos. Sci. 62:88-106.

Wood, R. 2005. Drizzle in stratiform boundary layer clouds. Part I: Vertical and horizontal structure. J. Atmos. Sci. 62:3011-3033.

Wood, R. 2007. Cancellation of aerosol indirect effects in marine stratocumulus through cloud thinning. J. Atmos. Sci. 64:2657-2669.

Wood, R., and C. S. Bretherton. 2006. On the relationship between stratiform low cloud cover and lower tropospheric stability. J. Climate 19:6425-6432.

Wood, R., D. W. Johnson, S. R. Osborne et al. 2000. Boundary layer and aerosol evolution during the third Lagrangian experiment of ACE-2. Tellus 52B:401-422.

Zhang, M. H., W. Y. Lin, S. A. Klein et al. 2005. Comparing clouds and their seasonal variations in 10 atmospheric general circulation models with satellite measurements. J. Geophys. Res. 110:D15S02.

Zhuang, L. Z., and B. J. Huebert. 1996. Lagrangian analysis of the total ammonia budget during Atlantic stratocumulus transition experiment marine aerosol and gas exchange. J. Geophys. Res. 101:4341-4350. 